\title{
Quaternion-Based Backstepping Control of Relative Attitude in a Spacecraft Formation
}

\author{
Raymond Kristiansen, Per Johan Nicklasson and Jan Tommy Gravdahl
}

\begin{abstract}
-
In this paper we present a solution to the problem of controlling relative attitude in a leader-follower spacecraft formation, with focus on the rotation path for the follower spacecraft. Mathematical models of relative attitude kinematics and dynamics are presented, and a nonlinear control law designed by use of integrator backstepping is used to control the relative attitude in the formation. The resulting control law is proved to result in asymptotically stable equilibrium points in the closed loop system, and by a convenient choice of backstepping variables, it is ensured that a proper rotation path is used. Simulation results for a leader-follower spacecraft formation are presented to illustrate the performance of the presented control law.
\end{abstract}

\section{INTRODUCTION}

\section{A. Background}

Spacecraft flying in formation are revolutionizing our way of performing space-based operations, and brings out several advantages in space mission accomplishment, as well as new opportunities and applications for such missions. The concept makes the way for new and better applications in space industry, such as improved monitoring of the Earth and its surrounding atmosphere, geodesy, deep-space imaging and exploration and even in-orbit spacecraft servicing and maintenance. The replacement of traditionally large and complex spacecraft with an array of simpler microsatellites introduces a multitude of advantages regarding mission cost and performance. However, the advantages of using spacecraft formations come at a cost of increased complexity and technological challenges. Formation flying introduces a control problem with strict and time-varying boundaries on spacecraft reference trajectories, and requires detailed knowledge and tight control of relative distances and velocities for participating spacecraft.

\section{B. Previous Work}

Synchronized control of relative attitude in spacecraft formations has received increased attention over the last years. State feedback tracking control laws for relative position and attitude were developed in [1], [2], and these solutions were proved to result in exponentially stable equilibrium points in the closed loop system. Nonlinear attitude tracking control of spacecraft formation was presented in [3], with an attitude parameter-independent open-loop solution based on

R. Kristiansen and P. J. Nicklasson are with the Department of Space technology, Narvik University College N-8515 Narvik, Norway. E-mail: \{rayk,pjn\}@hin.no.; J. T. Gravdahl is with the Department of Engineering Cybernetics, Norwegian University of Science and Technology, N7034 Trondheim, Norway. E-mail: tommy.gravdahl@itk.ntnu.no. a method for computing a multiaxis target tracking trajectory. A method for control of relative attitude in spacecraft formations using behavior-based control was presented in [4], where it was also showed how to rotate a group of spacecraft while maintaining the relative attitude. A method for coordinated attitude control by state feedback for the case where the leader satellite is not able to follow its reference attitude trajectory was proposed in [5], where the follower satellite is commanded to follow a combination of its reference attitude and the leader satellite attitude. In [6], a nonlinear tracking controller for both relative position and attitude was presented, including an adaptation law to account for unknown mass and inertia parameters of the spacecraft. The controller ensures global asymptotic convergence of position and velocity errors; the proof relies on a standard signal-chasing analysis and Barbalat's lemma. Semiglobal asymptotic convergence of relative position and attitude errors was proved in [7] for an adaptive output feedback controller using relative position only, tracing the steps of [6]. A scheme for attitude synchronization in a leader-follower spacecraft formation using attitude feedback only was presented in [8]. The controller was designed using vectorial backstepping, and the solution comprises two nonlinear observers for estimation of angular velocities. It was showed that the attitude error rotation matrix converges to the identity matrix in the closed loop system. An observercontroller structure for attitude synchronization of a leaderfollower spacecraft formation was also presented in [9], where the equilibrium points of the closed loop system was proved to be UGAS by an application of the Matrosov theorem.

\section{Contribution}

As described above, several approaches have been used to synchronize attitude of spacecraft flying in formation. Both state feedback and output feedback solutions have been presented, and the controllers have attractive properties with respect to stability. However, few solutions focus on power consumption and optimality of spacecraft rotations. As the spacecraft formation flying concept opens up for use of smaller spacecraft, there are increasing demands regarding weight, onboard equipment and low power consumption. Especially, for small spacecraft with limited space for energygenerating equipment such as solar panels, the possibility to control the spacecraft attitude optimal in terms of rotation path and power consumption is of utmost importance. To this end, we propose a control solution for regulation of relative attitude in a leader-follower spacecraft formation, focusing on optimality in rotation path, resulting in reduced power 
consumption. The solution is a quaternion-based controller designed by use of integrator backstepping, and is based on extending earlier works in [10], [11] on single-satellite attitude control. By combining the integrator backstepping concept and the redundant four-parameter attitude representation given by quaternions, we present a controller for relative attitude in a leader-follower spacecraft formation which results in asymptotically stable equilibrium points in the closed loop system. Our choice of backstepping design variables gives the advantage that the closest equilibrium point with respect to rotation path is always chosen when a given attitude change is commanded.

The rest of the paper is organized as follows: Section II defines the different reference frames used and presents the mathematical models of relative attitude dynamics and kinematics in a leader-follower spacecraft formation. The controller design is performed in Section III, and simulation results of a system with the derived controller are presented in Section IV. Concluding remarks are given in Section V.

\section{Modelling}

In this section, we formulate the satellite formation problem. We describe the different reference coordinate frames, together with the necessary coordinate transformations, that form the basis for our system, before we present models of the relative attitude kinematics and dynamics in a leaderfollower spacecraft formation.

\section{A. Cartesian Coordinate Frames}

The coordinate reference frames used throughout the paper are shown in Fig. 1 and defined as follows:

1) Earth Centered Inertial (ECI) Frame: This frame is denoted $\mathcal{F}_{i}$, and has its origin located in the center of the Earth. Its $z$ axis is directed along the rotation axis of the Earth towards the celestial north pole, the $x$ axis is directed towards the vernal equinox, and finally the direction of the $y$ axis completes a right handed orthogonal frame.

2) Leader Orbit Reference Frame: The leader orbit frame, denoted $\mathcal{F}_{l}$, has its origin located in the center of mass of the leader spacecraft. The $\mathbf{e}_{r}$ axis in the frame is parallel to the vector $\mathbf{r}_{l}$ pointing from the center of the Earth to the spacecraft, and the $\mathbf{e}_{h}$ axis is parallel to the orbit momentum vector, which points in the orbit normal direction. The $\mathbf{e}_{\theta}$ axis completes the right-handed orthogonal frame. The basis vectors of the frame can be defined as

$$
\mathbf{e}_{r}=\frac{\mathbf{r}_{l}}{r_{l}}, \quad \mathbf{e}_{\theta}=\mathbf{e}_{h} \times \mathbf{e}_{r} \quad \text { and } \quad \mathbf{e}_{h}=\frac{\mathbf{h}}{h},
$$

where $\mathbf{h}=\mathbf{r}_{l} \times \dot{\mathbf{r}}_{l}$ is the angular momentum vector of the orbit, and $h=|\mathbf{h}|$.

3) Follower Orbit Reference Frame: This frame has its origin in the center of mass of the follower spacecraft, and is denoted $\mathcal{F}_{f}$. The vector pointing from the center of the Earth to the center of the follower orbit frame is denoted $\mathbf{r}_{f}$. Its origin is specified by a relative orbit position vector $\mathbf{p}=\left[\begin{array}{lll}x & y & z\end{array}\right]^{\top}$ expressed in $\mathcal{F}_{l}$ frame components, as shown in Figure 1, and the frame unit vectors align with the basis vectors of $\mathcal{F}_{l}$. Accordingly,

$$
\mathbf{p}=\mathbf{r}_{f}-\mathbf{r}_{l}=x \mathbf{e}_{r}+y \mathbf{e}_{\theta}+z \mathbf{e}_{h} .
$$

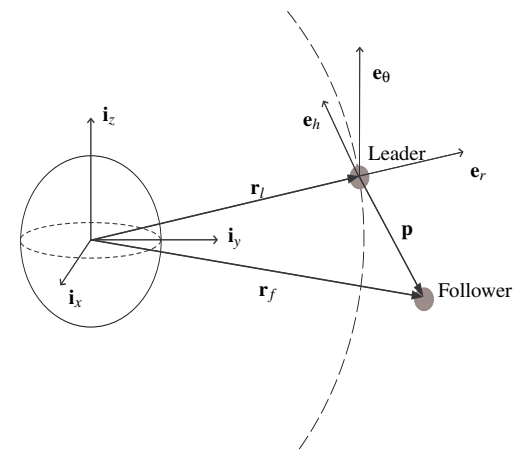

Fig. 1. Reference coordinate frames [12].

4) Body Reference Frames: For both the leader and the follower spacecraft, body reference frames are defined and denoted $\mathcal{F}_{b l}$ and $\mathcal{F}_{b f}$, respectively. These frames have, similar to the orbit frame, the origin located in the center of mass of the respective spacecraft, but the basis vectors are fixed in the spacecraft body and coincide with its principal axis of inertia.

\section{B. Coordinate Frame Transformations}

1) Rotation from ECI to Leader Orbit Frame: The rotation from the ECI frame to the leader orbit frame is dependent on the parameters of the leader spacecraft orbit, and can be expressed by three consecutive rotations. The total rotation matrix can be written ( $c f$. [13])

$$
\mathbf{R}_{i}^{l}=\mathbf{R}_{z, \omega+\nu} \mathbf{R}_{x, i} \mathbf{R}_{z, \Omega}
$$

where $\Omega$ is the right ascension of the ascending node of the orbit, $i$ is the inclination of the orbit, $v$ is the true anomaly of the leader orbit, and $\omega$ is the argument of perigee of the same. The sum of $v$ and $\omega$ represents the location of the spacecraft relative to the ascending node.

2) Body Frame Rotation: The rotation matrix describing rotations from an orbit frame to a body frame can be described by

$$
\mathbf{R}_{o}^{b}=\left[\begin{array}{lll}
\mathbf{c}_{1} & \mathbf{c}_{2} & \mathbf{c}_{3}
\end{array}\right]=\mathbf{I}+2 \eta \mathbf{S}(\varepsilon)+2 \mathbf{S}^{2}(\varepsilon)
$$

where the elements $\mathbf{c}_{i}$ are directional cosines, and

$$
\mathbf{q}=\left[\begin{array}{ll}
\eta & \varepsilon^{\top}
\end{array}\right]^{\top}
$$

are the Euler parameters, which satisfy the constraint

$$
\eta^{2}+\varepsilon^{\top} \varepsilon=1
$$

The matrix $\mathbf{S}(\cdot)$ is the cross product operator given by

$$
\mathbf{S}(\varepsilon)=\left[\begin{array}{ccc}
0 & -\varepsilon_{z} & \varepsilon_{y} \\
\varepsilon_{z} & 0 & -\varepsilon_{x} \\
-\varepsilon_{y} & \varepsilon_{x} & 0
\end{array}\right]
$$

when $\varepsilon=\left[\begin{array}{lll}\varepsilon_{x} & \varepsilon_{y} & \varepsilon_{z}\end{array}\right]^{\top}$. The inverse rotation is given by the complex conjugate of $\mathbf{q}$ as

$$
\overline{\mathbf{q}}=\left[\begin{array}{ll}
\eta & -\varepsilon^{\top}
\end{array}\right]^{\top}
$$


and the quaternion product is defined as (cf. [14])

$$
\mathbf{q}_{1} \otimes \mathbf{q}_{2} \triangleq\left[\begin{array}{c}
\eta_{1} \eta_{2}-\varepsilon_{1}^{\top} \varepsilon_{2} \\
\eta_{1} \varepsilon_{2}+\eta_{2} \varepsilon_{1}+\mathbf{S}\left(\varepsilon_{1}\right) \varepsilon_{2}
\end{array}\right] .
$$

\section{Relative Rotational Motion}

1) Attitude Kinematics: The time derivative of a rotation matrix $\mathbf{R}_{b}^{a}$ as in (4) can according to [14] be written as

$$
\dot{\mathbf{R}}_{b}^{a}=\mathbf{S}\left(\omega_{a, b}^{a}\right) \mathbf{R}_{b}^{a}=\mathbf{R}_{b}^{a} \mathbf{S}\left(\omega_{a, b}^{b}\right)
$$

where $\omega_{a, b}^{b}$ is the angular velocity of frame $b$ relative to frame $a$ represented in frame $b$ and $\mathbf{S}(\cdot)$ is the cross product operator described in (7). The kinematic differential equations for a spacecraft in its orbit frame can be found from (10) together with (5) as

$$
\dot{\mathbf{q}}_{s}=\left[\begin{array}{c}
\dot{\eta}_{s} \\
\dot{\varepsilon}_{s}
\end{array}\right]=\frac{1}{2}\left[\begin{array}{c}
-\varepsilon_{s}^{\top} \\
\eta_{s} \mathbf{I}+\mathbf{S}\left(\varepsilon_{s}\right)
\end{array}\right] \omega_{s, s b}^{s b}
$$

where $\omega_{s, s b}^{s b}$ is the angular velocity of the spacecraft body frame relative to the orbit frame, referenced in the body frame. The superscript/subscript $s$ is used in general to denote the spacecraft in question, so $s=l, f$ for the leader and follower spacecraft, respectively.

2) Attitude Dynamics: With the assumptions of rigid body movement, the dynamical model of a spacecraft can be derived from Euler's momentum equation as ( $c f$. [13])

$$
\begin{aligned}
\mathbf{J}_{s} \dot{\omega}_{i, s b}^{s b} & =-\mathbf{S}\left(\omega_{i, s b}^{s b}\right) \mathbf{J}_{s} \omega_{i, s b}^{s b}+\tau_{d s}^{s b}+\tau_{a s}^{s b} \\
\omega_{s, s b}^{s b} & =\omega_{i, s b}^{s b}+\omega_{o} \mathbf{c}_{2}
\end{aligned}
$$

where $\mathbf{J}_{s}$ is the spacecraft inertia matrix and $\omega_{i, s b}^{s b}$ is the angular velocity of the spacecraft body frame relative to the inertial frame, expressed in the body frame. The parameter $\omega_{o}$ is the orbit angular velocity, $\tau_{d}^{s b}$ is the total disturbance torque, $\tau_{a}^{s b}$ is the actuator torque, and $\mathbf{c}_{2}$ is the directional cosine vector from (4).

3) Relative Attitude: By expressing the relations in (11) and (12)-(13) for both the leader and the follower spacecraft, and utilising the quaternion product

$$
\mathbf{q}=\mathbf{q}_{f} \otimes \overline{\mathbf{q}}_{l}=\left[\begin{array}{c}
\eta_{f} \eta_{l}+\varepsilon_{f}^{\top} \varepsilon_{l} \\
\eta_{l} \varepsilon_{f}-\eta_{f} \varepsilon_{l}-\mathbf{S}\left(\varepsilon_{f}\right) \varepsilon_{l}
\end{array}\right]
$$

the relative attitude kinematics can be expressed as

$$
\dot{\mathbf{q}}=\left[\begin{array}{c}
\dot{\eta} \\
\dot{\varepsilon}
\end{array}\right]=\frac{1}{2}\left[\begin{array}{c}
-\varepsilon^{\top} \\
\eta \mathbf{I}+\mathbf{S}(\varepsilon)
\end{array}\right] \omega
$$

where

$$
\omega=\omega_{i, f b}^{f b}-\mathbf{R}_{l b}^{f b} \omega_{i, l b}^{l b}
$$

is the relative angular velocity between the leader body frame and the follower body frame. Moreover, from (16) the relative attitude dynamics can be expressed as

$$
\begin{aligned}
\mathbf{J}_{f} \dot{\boldsymbol{\omega}} & =\mathbf{J}_{f} \dot{\boldsymbol{\omega}}_{i, f b}^{f b}-\mathbf{J}_{f} \dot{\mathbf{R}}_{l b}^{f b} \omega_{i, l b}^{l b}-\mathbf{J}_{f} \mathbf{R}_{l b}^{f b} \dot{\boldsymbol{\omega}}_{i, l b}^{l b} \\
& =\mathbf{J}_{f} \dot{\boldsymbol{\omega}}_{i, f b}^{f b}-\mathbf{J}_{f} \mathbf{S}\left(\omega_{i, l b}^{f b}\right) \omega-\mathbf{J}_{f} \mathbf{R}_{l b}^{f b} \dot{\omega}_{i, l b}^{l b}
\end{aligned}
$$

where (10) and the facts that $\omega_{l b, f b}^{f b}=\omega$ and $\mathbf{S}(\mathbf{a}) \mathbf{b}=$ $-\mathbf{S}(\mathbf{b}) \mathbf{a}$ have been used. Insertion of (12), evaluated for both leader and follower, into (18) results in

$$
\begin{aligned}
\mathbf{J}_{f} \dot{\boldsymbol{\omega}} & +\mathbf{J}_{f} \mathbf{S}\left(\mathbf{R}_{l b}^{f b} \boldsymbol{\omega}_{i, l b}^{l b}\right) \omega-\mathbf{J}_{f} \mathbf{R}_{l b}^{f b} \mathbf{J}_{l}^{-1} \mathbf{S}\left(\omega_{i, l b}^{l b}\right) \mathbf{J}_{l} \omega_{i, l b}^{l b} \\
& +\mathbf{S}\left(\omega+\mathbf{R}_{l b}^{f b} \omega_{i, l b}^{l b}\right) \mathbf{J}_{f}\left(\omega+\mathbf{R}_{l b}^{f b} \omega_{i, l b}^{l b}\right)=\Upsilon_{d}+\Upsilon_{a}
\end{aligned}
$$

where

$$
\Upsilon_{d}=\tau_{d f}^{f b}-\mathbf{J}_{f} \mathbf{R}_{l b}^{f b} \mathbf{J}_{l}^{-1} \tau_{d l}^{l b}
$$

and

$$
\Upsilon_{a}=\tau_{a f}^{f b}-\mathbf{J}_{f} \mathbf{R}_{l b}^{f b} \mathbf{J}_{l}^{-1} \tau_{a l}^{l b}
$$

are the relative disturbance torques and relative actuator torques, respectively. The fourth term in (19) can be rewritten as

$$
\begin{aligned}
& \mathbf{S}\left(\omega+\mathbf{R}_{l b}^{f b} \omega_{i, l b}^{l b}\right) \mathbf{J}_{f}\left(\omega+\mathbf{R}_{l b}^{f b} \omega_{i, l b}^{l b}\right)= \\
&\left(\mathbf{S}\left(\mathbf{R}_{l b}^{f b} \omega_{i, l b}^{l b}\right)\right.\left.\mathbf{J}_{f}-\mathbf{S}\left(\mathbf{J}_{f}\left(\omega+\mathbf{R}_{l b}^{f b} \omega_{i, l b}^{l b}\right)\right)\right) \omega \\
&+ \mathbf{S}\left(\mathbf{R}_{l b}^{f b} \omega_{i, l b}^{l b}\right) \mathbf{J}_{f} \mathbf{R}_{l b}^{f b} \omega_{i, l b}^{l b}
\end{aligned}
$$

and accordingly, (19) can be written as

$$
\mathbf{J}_{f} \dot{\boldsymbol{\omega}}+\mathbf{C}(\omega) \omega+\mathbf{n}(\omega)=\Upsilon_{d}+\Upsilon_{a}
$$

where

$$
\begin{aligned}
\mathbf{C}(\omega)=\mathbf{J}_{f} \mathbf{S}\left(\mathbf{R}_{l b}^{f b} \omega_{i, l b}^{l b}\right)+ & \mathbf{S}\left(\mathbf{R}_{l b}^{f b} \omega_{i, l b}^{l b}\right) \mathbf{J}_{f} \\
& -\mathbf{S}\left(\mathbf{J}_{f}\left(\omega+\mathbf{R}_{l b}^{f b} \omega_{i, l b}^{l b}\right)\right)
\end{aligned}
$$

is skew-symmetric, $\mathbf{C}(\omega) \in S S(3)$, and

$$
\begin{aligned}
\mathbf{n}(\omega)=\mathbf{S}\left(\mathbf{R}_{l b}^{f b} \omega_{i, l b}^{l b}\right) & \mathbf{J}_{f} \mathbf{R}_{l b}^{f b} \omega_{i, l b}^{l b} \\
& -\mathbf{J}_{f} \mathbf{R}_{l b}^{f b} \mathbf{J}_{l}^{-1} \mathbf{S}\left(\omega_{i, l b}^{l b}\right) \mathbf{J}_{l} \omega_{i, l b}^{l b}
\end{aligned}
$$

is a nonlinear term.

\section{Controller Design}

Having established the mathematical models of relative attitude kinematics and dynamics in a leader-follower formation, we now present a solution to the control problem, using the integrator backstepping concept to design a control law based on quaternions. We also provide an analysis of the resulting stability properties, together with some remarks regarding the solution. Finally, we present some notes on the implementation of the control law.

\section{A. Integrator Backstepping}

To solve the control problem, a control law for regulating the relative attitude between the leader and follower spacecraft from an arbitrary initial quaternion $\mathbf{q}_{0}$ to a constant reference quaternion $\mathbf{q}_{d}=\left[\begin{array}{ll}\eta_{d} & \varepsilon_{d}^{\top}\end{array}\right]^{\top}$ is needed.

Step 1 The first step in integrator backstepping involves control of (15), and the first backstepping variable is chosen as

$$
\mathbf{z}_{1}=\left[\begin{array}{c}
1-|\tilde{\eta}| \\
\tilde{\varepsilon}
\end{array}\right]
$$


where $\tilde{\mathbf{q}}=\left[\tilde{\eta} \tilde{\varepsilon}^{\top}\right]^{\top}$ is given from the quaternion product

$$
\tilde{\mathbf{q}}=\mathbf{q}_{d} \otimes \overline{\mathbf{q}}=\left[\begin{array}{c}
\eta_{d} \eta+\varepsilon_{d}^{\top} \varepsilon \\
\eta \varepsilon_{d}-\eta_{d} \varepsilon-\mathbf{S}\left(\varepsilon_{d}\right) \varepsilon
\end{array}\right] .
$$

Perfect set-point control can be expressed in quaternion notation as [15]

$$
\mathbf{q}=\mathbf{q}_{d} \quad \Leftrightarrow \quad \tilde{\mathbf{q}}=\left[\begin{array}{c} 
\pm 1 \\
\mathbf{0}
\end{array}\right] .
$$

Similar to (15), the error kinematics can be expressed as

$$
\begin{aligned}
\dot{\tilde{\mathbf{q}}} & =\frac{1}{2}\left[\begin{array}{c}
-\tilde{\varepsilon}^{\top} \\
\tilde{\eta} \mathbf{I}+\mathbf{S}(\tilde{\varepsilon})
\end{array}\right] \tilde{\omega} \\
& =-\frac{1}{2}\left[\begin{array}{c}
-\tilde{\varepsilon}^{\top} \\
\tilde{\eta} \mathbf{I}+\mathbf{S}(\tilde{\varepsilon})
\end{array}\right] \omega
\end{aligned}
$$

since $\tilde{\omega}=\omega_{d}-\omega=-\omega$ where the desired relative angular velocity $\omega_{d}=\mathbf{0}$. A virtual control input is defined as

$$
\omega=\alpha_{1}+\mathbf{z}_{2}
$$

where $\alpha_{1}$ is a stabilizing function and $\mathbf{z}_{2}$ is a new state variable. This, together with (30) leaves the $\mathbf{z}_{1}$-system as

$$
\begin{aligned}
& \dot{\mathbf{z}}_{1}=\left[\begin{array}{c}
-\operatorname{sgn}(\tilde{\eta}) \dot{\tilde{\eta}} \\
\dot{\tilde{\varepsilon}}
\end{array}\right] \\
& =-\frac{1}{2}\left[\begin{array}{c}
\operatorname{sgn}(\tilde{\eta}) \tilde{\varepsilon}^{\top} \\
\tilde{\eta} \mathbf{I}+\mathbf{S}(\tilde{\varepsilon})
\end{array}\right] \omega \\
& =-\frac{1}{2} \mathbf{G}^{\top}(\tilde{\mathbf{q}})\left(\alpha_{1}+\mathbf{z}_{2}\right)
\end{aligned}
$$

where

$$
\mathbf{G}^{\top}(\tilde{\mathbf{q}})=\left[\begin{array}{c}
\operatorname{sgn}(\tilde{\eta}) \tilde{\varepsilon}^{\top} \\
\tilde{\eta} \mathbf{I}+\mathbf{S}(\tilde{\varepsilon})
\end{array}\right] .
$$

It can be shown that

$$
\mathbf{G}(\tilde{\mathbf{q}}) \mathbf{z}_{1}=\mathbf{0} \quad \Leftrightarrow \quad \operatorname{sgn}(\tilde{\eta}) \tilde{\varepsilon}=\mathbf{0}
$$

and the signum function $\operatorname{sgn}(\mathrm{x})$ is therefore defined nonzero as

$$
\operatorname{sgn}(\mathrm{x})=\left\{\begin{array}{rr}
-1, & x<0 \\
1, & x \geq 0
\end{array}\right.
$$

to avoid a singularity when $\tilde{\eta}=0$. A Lyapunov Function Candidate (LFC) is chosen as

$$
\begin{aligned}
& V_{1}=\mathbf{z}_{1}^{\top} \mathbf{z}_{1} \\
& \dot{V}_{1}=2 \mathbf{z}_{1}^{\top} \dot{\mathbf{z}}_{1}=-\mathbf{z}_{1}^{\top} \mathbf{G}^{\top}(\tilde{\mathbf{q}})\left(\alpha_{1}+\mathbf{z}_{2}\right) .
\end{aligned}
$$

Furthermore, the stabilizing function $\alpha_{1}$ is chosen as

$$
\alpha_{1}=\mathbf{K}_{1} \mathbf{G}(\tilde{\mathbf{q}}) \mathbf{z}_{1}
$$

where $\mathbf{K}_{1}=\mathbf{K}_{1}^{\top}>0$ is a feedback gain matrix. Inserting this into the LFC in (39) yields

$$
\dot{V}_{1}=-\mathbf{z}_{1}^{\top} \mathbf{G}^{\top} \mathbf{K}_{1} \mathbf{G} \mathbf{z}_{1}-\mathbf{z}_{1}^{\top} \mathbf{G}^{\top} \mathbf{z}_{2}
$$

where the argument of the matrix $\mathbf{G}(\tilde{\mathbf{q}})$ is left out for readability. It should be noted that $\mathbf{G}^{\top} \mathbf{K}_{1} \mathbf{G}$ is a symmetric positive semidefinite matrix. The $\mathbf{z}_{1}$-system from (34) can be written accordingly as

$$
\begin{aligned}
\dot{\mathbf{z}}_{1} & =-\frac{1}{2} \mathbf{G}^{\top}\left(\alpha_{1}+\mathbf{z}_{2}\right) \\
& =-\frac{1}{2} \mathbf{G}^{\top} \mathbf{K}_{1} \mathbf{G} \mathbf{z}_{1}-\frac{1}{2} \mathbf{G}^{\top} \mathbf{z}_{2} .
\end{aligned}
$$

Step 2 For the second step, (31) is differentiated to express the $\mathbf{z}_{2}$-dynamics as

$$
\dot{\mathbf{z}}_{2}=\dot{\omega}-\dot{\alpha}_{1}
$$

and insertion of (23) leaves

$$
\begin{aligned}
\mathbf{J}_{f} \dot{\mathbf{z}}_{2} & =\mathbf{J}_{f} \dot{\boldsymbol{\omega}}-\mathbf{J}_{f} \dot{\alpha}_{1} \\
& =\Upsilon_{d}+\Upsilon_{a}-\mathbf{C}(\omega) \omega-\mathbf{n}(\omega)-\mathbf{J}_{f} \dot{\alpha}_{1} .
\end{aligned}
$$

A second LFC can now be expressed as

$$
V_{2}=V_{1}+\frac{1}{2} \mathbf{z}_{2}^{\top} \mathbf{J}_{f} \mathbf{z}_{2}
$$

and hence

$$
\begin{aligned}
\dot{V}_{2} & =\dot{V}_{1}+\mathbf{z}_{2}^{\top} \mathbf{J}_{f} \dot{\mathbf{z}}_{2} \\
& =\dot{V}_{1}+\mathbf{z}_{2}^{\top}\left[\Upsilon_{d}+\Upsilon_{a}-\mathbf{C}(\omega) \omega-\mathbf{n}(\omega)-\mathbf{J}_{f} \dot{\alpha}_{1}\right] .
\end{aligned}
$$

Assume that the leader spacecraft is perfectly controlled in its orbit, so that $\tau_{a l}^{l b}=-\tau_{d l}^{l b}$, and that the disturbance torque $\tau_{d f}^{f b}$ can be measured or estimated. Then, choosing the actuator torque as

$$
\tau_{a f}^{f b}=-\mathbf{K}_{2} \mathbf{z}_{2}+\mathbf{G} \mathbf{z}_{1}+\mathbf{C}(\omega) \omega+\mathbf{n}(\omega)+\mathbf{J}_{f} \dot{\alpha}_{1}-\tau_{d f}^{f b}
$$

where $\mathbf{K}_{2}=\mathbf{K}_{2}^{\top}>0$ is the feedback gain matrix for the $\mathbf{z}_{2}$ system, leaves the LFC as

$$
\begin{aligned}
\dot{V}_{2} & =\dot{V}_{1}+\mathbf{z}_{2}^{\top}\left[-\mathbf{K}_{2} \mathbf{z}_{2}+\mathbf{G z}_{1}\right] \\
& =-\mathbf{z}_{1}^{\top} \mathbf{G}^{\top} \mathbf{K}_{1} \mathbf{G} \mathbf{z}_{1}-\mathbf{z}_{2}^{\top} \mathbf{K}_{2} \mathbf{z}_{2} \leq \mathbf{0}
\end{aligned}
$$

and the closed-loop dynamics as

$$
\begin{aligned}
\dot{\mathbf{z}}_{1} & =-\frac{1}{2} \mathbf{G}^{\top} \mathbf{K}_{1} \mathbf{G} \mathbf{z}_{1}-\frac{1}{2} \mathbf{G}^{\top} \mathbf{z}_{2} \\
\mathbf{J}_{f} \dot{\mathbf{z}}_{2} & =-\mathbf{K}_{2} \mathbf{z}_{2}+\mathbf{G} \mathbf{z}_{1} .
\end{aligned}
$$

\section{B. Stability Properties}

The stability properties of the closed loop system given by (53)-(54) follows from (47) and (52). From (47) it is seen that $V_{2}\left(\mathbf{z}_{1}, \mathbf{z}_{2}\right)>0, V_{2}(0)=0$ and $V_{2}\left(\mathbf{z}_{1}, \mathbf{z}_{2}\right) \rightarrow \infty$ as $\left(\mathbf{z}_{1}, \mathbf{z}_{2}\right) \rightarrow \infty$. Hence, Lyapunovs direct method [16] ensures stability and therefore boundedness of $\mathbf{G} \mathbf{z}_{1}, \mathbf{z}_{2}$. Moreover, it is recognized from (52) that $\dot{V}_{2}=0$ implies $\left(\mathbf{G z}_{1}, \mathbf{z}_{2}\right)=(\mathbf{0 , 0})$, and furthermore from (6), (31), (36) and (40) that $\tilde{\varepsilon}=\mathbf{0}$, $\tilde{\eta}= \pm 1$, and $\omega=\mathbf{0}$. Hence, from invoking standard Lyapunov theorems [16], it follows that both of the equilibrium points $\tilde{\mathbf{q}}=\left[ \pm 1 \mathbf{0}^{\top}\right]^{\top}$ are asymptotically stable (AS), and it follows from (28) that $\mathbf{q} \rightarrow \mathbf{q}_{d}$ as $t \rightarrow \infty$.

Remark 1: If the first backstepping variable in (26) is chosen as

$$
\mathbf{z}_{1}=\left[\begin{array}{c}
1-\tilde{\eta} \\
\tilde{\varepsilon}
\end{array}\right]
$$

the equilibrium point $\tilde{\mathbf{q}}=\left[\begin{array}{ll}1 & \mathbf{0}^{\top}\end{array}\right]^{\top}$ will be asymptotically stable, while $\tilde{\mathbf{q}}=\left[\begin{array}{ll}-1 & \mathbf{0}^{\top}\end{array}\right]^{\top}$ will be unstable. Hence, the 
scalar part of the quaternion must always be regulated to the stable point where $\tilde{\eta}=1$, even if a rotation to the point where $\tilde{\eta}=-1$ requires less power. A discussion concerning this can be found in [17]. When both equilibrium points are asymptotically stable, the relative attitude can be regulated to the closest equilibrium point with respect to rotation path, which will imply less use of energy.

Remark 2: We stress that the asymptotic stability properties for the equilibrium points are only local in $S O(3)$. Since the unit quaternion parameter set is redundant, a given physical attitude for a rigid body will have two mathematical representations, where one of these includes a rotation of $2 \pi$ about an axis relative to the other. This is the advantage that is exploited by our choice of backstepping variables, to render both equilibrium points asymptotically stable, as mentioned in Remark 1. Based on the physical nature of the problem, it might be appealing to think that the equilibrium point set $\tilde{\mathbf{q}}=\left[ \pm 1 \mathbf{0}^{\top}\right]^{\top}$ has global stability properties, but this is however not the case, at least not in $S O(3)$. A discussion concerning this point can be found in [18].

Remark 3: The second term of the control torque equation (50) includes the matrix $\mathbf{G}(\tilde{\mathbf{q}})$, which can be interpreted as a state dependent feedback gain matrix. This implies that as the quaternion error increases, the gain will do the same.

Remark 4: The asymptotic stability property of the equilibrium points is a result of the assumption of known disturbance torque $\tau_{d f}^{f b}$ and a perfectly controlled leader. If this assumption is relaxed to unknown, but bounded, disturbance torques and leader motion, it can be shown that the stability property will degenerate to uniform practical stability ( $c f$. [19], [20]). That is, the state errors in the closed-loop system can be proved to converge from any initial conditions to a ball in close vicinity of the origin in a stable way, and this ball can be diminished arbitrarily by increasing the gains in the control law. However, the stronger assumption is used in this paper to better illustrate the point of utilizing the quaternion redundancy, as mentioned in Remark 1.

\section{Implementation}

The control law given by (50) contains the expression $\dot{\alpha}_{1}$ that involves time derivatives of the states, and this should be avoided when the control law is implemented. A possible solution is to use the model equations as a sort of smooth differentiators, instead of differentiating the measurements directly. The time differentiation of (40) can be performed as

$$
\dot{\alpha}_{1}=\mathbf{K}_{1}\left[\dot{\mathbf{G}}(\tilde{\mathbf{q}}) \mathbf{z}_{1}+\mathbf{G}(\tilde{\mathbf{q}}) \dot{\mathbf{z}}_{1}\right]
$$

where $\dot{\mathbf{z}}_{1}$ can be found from (33). Moreover, $\dot{\mathbf{G}}(\tilde{\mathbf{q}})$ can be expressed as

$$
\dot{\mathbf{G}}(\tilde{\mathbf{q}})=\frac{\partial \mathbf{G}}{\partial \tilde{\eta}} \dot{\tilde{\eta}}+\frac{\partial \mathbf{G}}{\partial \tilde{\varepsilon}} \dot{\tilde{\varepsilon}}=\left[\begin{array}{c}
\operatorname{sgn}(\tilde{\eta}) \dot{\tilde{\varepsilon}}^{\top} \\
\dot{\tilde{\eta}} \mathbf{I}+\mathbf{S}(\dot{\tilde{\varepsilon}})
\end{array}\right]^{T} .
$$

The expressions for $\dot{\tilde{\eta}}$ and $\dot{\tilde{\varepsilon}}$ as presented in (30) can now be inserted to remove the time differentiated states in the controller.
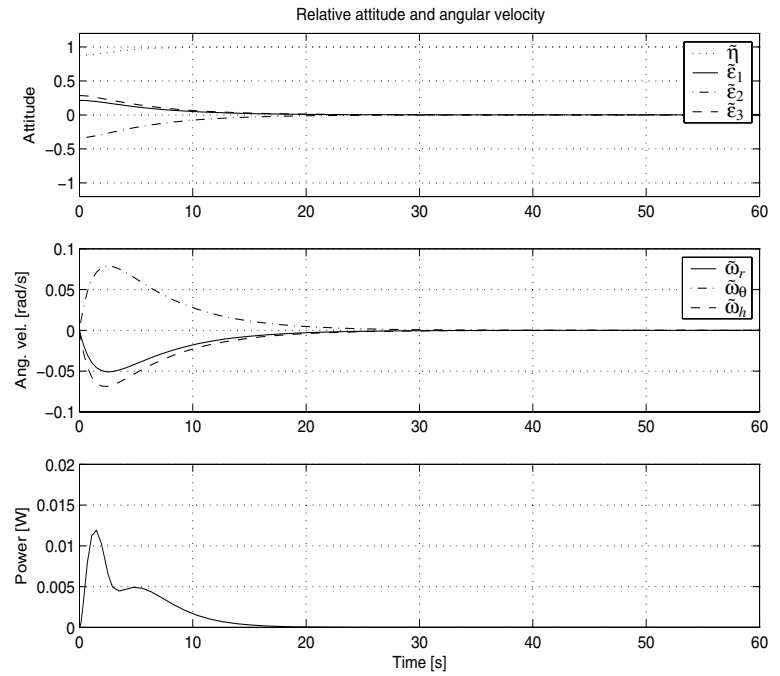

Fig. 2. Simulation result of a spacecraft formation using quaternion-based backstepping, small angle deviation.

\section{Simulations}

In this section, simulation results are presented to illustrate the performance of the presented control law. In the simulations, the orbital perturbations are assumed to be perfectly known or estimated based on measurements. Both the leader and the follower spacecraft have moments of inertia given as $\mathbf{J}=\operatorname{diag}\left\{\begin{array}{lll}4.350 & 4.3370 & 3.6640\end{array}\right\} \mathrm{kgm}^{2}$. The leader is assumed to follow an equatorial orbit with a perigee altitude of $250 \mathrm{~km}$ and eccentricity $e=0.3$. The follower is assumed to have available continuous torque about all body axes, with a maximum torque of $0.4 \mathrm{Nm}$. In all the simulations, the controller gains $\mathbf{K}_{1}=0.05 \mathbf{I}$ and $\mathbf{K}_{2}=4 \mathbf{I}$ have been used.

\section{A. Results}

The simulation results of a spacecraft formation where the follower uses the backstepping controller (50) are presented in the following. Fig. 2 shows the relative attitude error in terms of quaternions, the relative angular velocity error and power consumption of the follower when the initial relative attitude between the leader and follower spacecraft is $\Theta_{i}=$ $\left[15^{\circ}-45^{\circ} 30^{\circ}\right]^{\top}$, where $\Theta$ denotes the Euler angles for the axes $\mathbf{e}_{r}, \mathbf{e}_{\theta}$ and $\mathbf{e}_{h}$, respectively, and the desired relative attitude is $\Theta_{d}=\left[\begin{array}{lll}0^{\circ} & 0^{\circ} & 0^{\circ}\end{array}\right]^{\top}$. This corresponds to the quaternion values $\mathbf{q}_{i}=\left[\begin{array}{llll}0.8718 & 0.2147 & -0.3353 & 0.2853\end{array}\right]^{\top}$ and $\mathbf{q}_{d}=$ $\left[\begin{array}{llll} \pm 1 & 0 & 0 & 0\end{array}\right]^{\top}$. The desired relative attitude is reached in approximately 20 seconds, and the power consumption over one orbit is $0.1426 \mathrm{Ws}$, with peak consumption $0.0120 \mathrm{~W}$.

Fig. 3 shows the relative attitude error, the relative angular velocity error and the power consumption of the follower spacecraft when the relative initial attitude in the formation is $\Theta_{i}=\left[-75^{\circ}-175^{\circ} 70^{\circ}\right]^{\top}$ and the desired relative attitude is $\Theta_{d}=\left[\begin{array}{lll}0^{\circ} & 0^{\circ} & 0^{\circ}\end{array}\right]^{\top}$. This corresponds to the quaternion values $\mathbf{q}_{i}=\left[\begin{array}{llll}-0.3772 & -0.4329 & 0.6645 & 0.4783\end{array}\right]^{\top}$ and $\mathbf{q}_{d}=$ $\left[\begin{array}{llll} \pm 1 & 0 & 0 & 0\end{array}\right]^{\top}$. The desired relative attitude is reached in approximately 25 seconds, and the power consumption over one orbit is $0.9315 \mathrm{Ws}$, with a peak power consumption of $0.0458 \mathrm{~W}$. Note that the relative attitude error converges to 

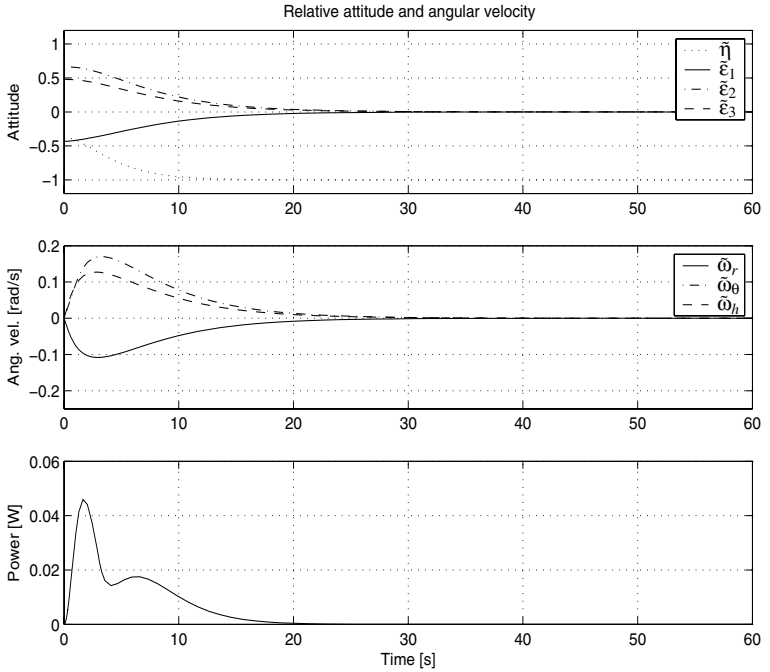

Fig. 3. Simulation result of a spacecraft formation using quaternion-based backstepping, large angle deviation.
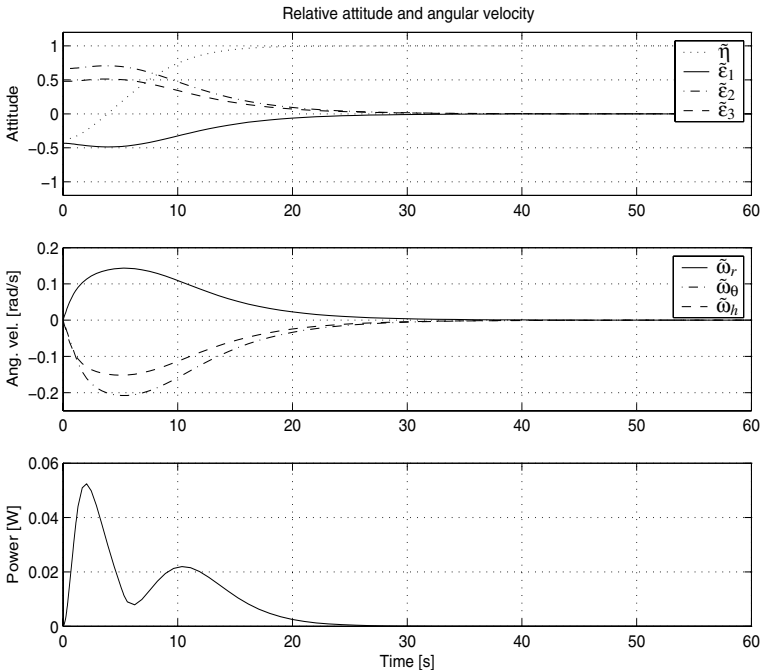

Fig. 4. Simulation result of a spacecraft formation using quaternion-based backstepping, large angle deviation, suboptimal rotation.

the equilibrium point $\tilde{\mathbf{q}}=\left[\begin{array}{ll}-1 & \mathbf{0}^{\top}\end{array}\right]^{\top}$, as opposed to Fig. 2 where the resulting relative attitude error is $\tilde{\mathbf{q}}=\left[\begin{array}{ll}1 & \mathbf{0}^{\top}\end{array}\right]^{\top}$.

This latter simulation result can be compared with the one given in Fig. 4, where a controller derived by the same approach, but with $\mathbf{z}_{1}$ given by (55) is used. The initial and desired relative attitudes are the same as in Fig. 3, but since $\tilde{\mathbf{q}}=\left[\begin{array}{ll}1 & \mathbf{0}^{\top}\end{array}\right]^{\top}$ is the only stable equilibrium point, the controller forces the relative attitude in the formation towards this. The result is an increased settling time of about $20 \%$ to approximately 30 seconds, and the power consumption goes up by approximately $32 \%$ to $1.234 \mathrm{Ws}$, and peak power increases with approximately $14 \%$ to $0.0522 \mathrm{~W}$.

\section{CONCLUSION}

In this paper we have presented a solution to the problem of controlling relative attitude in a leader-follower spacecraft formation, with focus on optimality in rotation path for the follower spacecraft. Mathematical models of relative attitude kinematics and dynamics were presented, and a nonlinear control law designed by using integrator backstepping was derived to control the relative attitude in the formation. The resulting control law was proved to result in asymptotically stable equilibrium points in the closed loop system, and by a convenient choice of backstepping variables, it was ensured that a proper rotation path was used in terms of optimality. Finally, simulation results for a leader-follower spacecraft formation were presented to illustrate the performance of the presented control law.

\section{REFERENCES}

[1] P. K. C. Wang and F. Y. Hadaegh, "Coordination and control of multiple microspacecraft moving in formation," $J$. of Astronautical Sciences, vol. 44, no. 3, pp. 315-355, 1996.

[2] P. K. C. Wang, F. Y. Hadaegh, and K. Lau, "Synchronized formation rotation and attitude control of multiple free-flying spacecraft," AIAA J. of Guidance, Control and Dynamics, vol. 22, no. 1, pp. 28-35, 1999.

[3] M. R. Long and C. D. Hall, "Attitude tracking control for spacecraft formation flying," in Presented at The Flight Mechanics Symposium, Goddard Space Flight Center, 1999.

[4] J. Lawton and R. W. Beard, "Elementary attitude formation maneuver via leader-following and behaviour-based control," in Proc. of the AIAA Guidance, Navigation and Control Conf., Denver, CO, 2000.

[5] W. Kang and H. Yeh, "Co-ordinated attitude control of multi-satellite systems," International J. of Robust and Nonlinear Control, vol. 12, pp. 185-205, 2001.

[6] H. Pan and V. Kapila, "Adaptive nonlinear control for spacecraft formation flying with coupled translational and attitude dynamics," in Proc. of the Conf. on Decision and Control, Orlando, FL, 2001.

[7] H. Wong, H. Pan, and V. Kapila, "Output feedback control for spacecraft formation flying with coupled translation and attitude dynamics," in Proc. of the American Control Conf., Portland, OR, 2005.

[8] A. K. Bondhus, K. Y. Pettersen, and J. T. Gravdahl, "Leader/follower synchronization of satellite attitude without angular velocity measurements," in Proc. of the IEEE Conf. on Decision and Control, Seville, Spain, 2005.

[9] T. R. Krogstad, J. T. Gravdahl, and R. Kristiansen, "Coordinated control of satellites: The attitude case," in Proc. of the International Astronautical Congress, Fukuoka, Japan, 2005.

[10] R. Kristiansen and P. J. Nicklasson, "Satellite attitude control by quaternion-based backstepping," in Proc. of the American Control Conf., Portland, Oregon, 2005.

[11] R. Kristiansen, P. J. Nicklasson, and J. T. Gravdahl, "Satellite attitude tracking by quaternion-based backstepping," in Proc. of the IFAC World Congress, Prague, Czech Republic, 2005.

[12] H. Schaub and J. L. Junkins, Analytical Mechanics of Space Systems, ser. AIAA Education Series. Reston, VA: American Institute of Aeronautics and Astronautics, 2003.

[13] M. J. Sidi, Spacecraft Dynamics and Control. New York: Cambridge University Press, 1997.

[14] O. Egeland and J. T. Gravdahl, Modeling and Simulation for Automatic Control. Trondheim, Norway: Marine Cybernetics, 2002.

[15] O.-E. Fjellstad, "Control of unmanned underwater vehicles in six degrees of freedom," Ph.D. dissertation, Department of Engineering Cybernetics, Norwegian University of Science and Technology, Trondheim, Norway, 1994.

[16] H. K. Khalil, Nonlinear Systems, third edition. Upper Saddle River, New Jersey, USA: Pearson Education International Inc., 2002.

[17] J. T.-Y. Wen and K. Kreutz-Delgado, "The attitude control problem," IEEE Transactions on Automatic Control, vol. 36, no. 10, pp. 11481162, 1991.

[18] S. P. Bhat and D. S. Bernstein, "A topological obstruction to continuous global stabilization of rotational motion and the unwinding phenomenon," Systems \& Control Letters, vol. 39, pp. 63-70, 2000.

[19] R. Kristiansen, A. Loría, A. Chaillet, and P. J. Nicklasson, "Output feedback control of relative translation in a leader-follower spacecraft formation," in ser. Lecture Notes in Control and Information Sciences, K. Y. Pettersen, J. T. Gravdahl, and H. Nijmeijer, Eds. Springer Verlag, 2006.

[20] A. Chaillet and A. Loría, "Uniform semiglobal practical asymptotic stability for non-autonomous cascaded systems and applications," 2006, http://arxiv.org/PS_cache/math/pdf/0503/0503039.pdf. 